\title{
Intraocular vision-improving devices in age-related macular degeneration
}

\author{
Andrzej Grzybowski ${ }^{1,2 \#}$, Jin Wang $^{3 \#}$, Feifei Mao ${ }^{4}$, Dabo Wang ${ }^{5}$, Ningli Wang ${ }^{3,6,7,8}$ \\ ${ }^{1}$ Department of Ophthalmology, University of Warmia and Mazury, Olsztyn, Poland; ${ }^{2}$ Institute for Research in Ophthalmology, Foundation for \\ Ophthalmology Development, Poznan, Poland; ${ }^{3}$ Department of Ophthalmology, Beijing Tongren Hospital, Capital Medical University, Beijing, \\ China; ${ }^{4}$ Department of Ophthalmology, Beijing DiTan Hospital, Capital Medical University, Beijing, China; ${ }^{5}$ Department of Ophthalmology, The \\ Affiliated Hospital of Qingdao University, Qingdao, China; ${ }^{6}$ Beijing Institute of Ophthalmology, Beijing, China; ${ }^{7}$ Beijing Tongren Eye Center, \\ Beijing, China; ${ }^{8}$ Beijing Key Laboratory of Ophthalmology and Visual Sciences, Beijing, China \\ Contributions: (I) Conception and design: A Grzybowski, J Wang, N Wang; (II) Administrative support: A Grzybowski, F Mao, D Wang; (III) \\ Provision of study materials or patients: A Grzybowski, J Wang, N Wang; (IV) Collection and assembly of data: A Grzybowski, J Wang; (V) Data \\ analysis and interpretation: A Grzybowski, J Wang; (VI) Manuscript writing: All authors; (VII) Final approval of manuscript: All authors. \\ "These authors contributed equally to this work. \\ Correspondence to: Prof. Ningli Wang, MD, PhD. Beijing Tongren Eye Center, Beijing Tongren Hospital, Capital Medical University, Beijing \\ Ophthalmology, and Visual Science Key Lab, No. 1 Dong Jiao Min Xiang Street, Dongcheng District, Beijing, China. Email: wningli@vip.163.com.
}

\begin{abstract}
Age-related macular degeneration (AMD) is a leading cause of blindness, especially in western countries, and will substantially burden society and the world's health care system. Patients with late AMD often accompany a progressive loss of central vision, which will heavily influence the quality of life and associated with increased risk of functional disability. The principal visual rehabilitation methods with low-vision magnifiers, such as hand or stand magnifiers, spectacles, and closed-circuit television, were cumbersome to use and cosmetically burdensome. Therefore, the development of intraocular visionimproving devices has become an attractive alternative to extraocular visual aids, and better life quality improvement has been reached among AMD patients. To evaluate each device's safety and efficacy based on current research, we searched the Cochrane Library, PubMed using pre-reported search terms and keywords combined with both Mesh term and text words. We explored randomized clinical controlled trials, cohort studies, and case serial reports and summarizes three aspects: visual outcomes, safety outcome, and quality of life outcomes. There are four types of devices recommended for AMD patients illustrating in this article: an implantable miniature telescope (IMT), IOL-VIP System, EyeMax Mono, and Scharioth macula lens (SML). There is no doubt that these technological advancements would bring new hope for AMD patients. However, the lack of randomized controls, limited follow-up duration, and various visual acuity (VA) measurements in different studies would be difficult than IOL devices.
\end{abstract}

Keywords: Intraocular devices; implantable miniature telescope (IMT); EyeMax Mono; IOL-VIP system; Scharioth macula lens (SML)

Submitted Aug 12, 2020. Accepted for publication Nov 09, 2020.

doi: $10.21037 / \mathrm{atm}-20-5851$

View this article at: http://dx.doi.org/10.21037/atm-20-5851

\section{Introduction}

Age-related macular degeneration (AMD), a leading cause of visual impairment in developed countries, affects the retina's macular region, causing severe and progressive loss of central vision (1). On a global scale, the number of people with AMD is expected to be 200 million by 2020 and increasing to 300 million by 2040 (2). The estimated prevalence of late AMD in the three extensive populationbased studies in European white people over 85 years old was $13.1 \%$ (3), and the 15 -year incidence of late AMD was 
$6.8 \%$ (4). However, in Asia and Africa, the prevalence of late AMD was only $0.1-0.3 \%$ (2), and the 6-year incidence for early and late AMD is $4.2 \%$ and $0.2 \%$, respectively (5). AMD occurs commonly in developed countries, potentially indicating that progression in demographic composition like aging, might play roles in the prevalence of the disease (6). Although AMD's prevalence was lowest currently in Asia, the population still accounts for more than $60 \%$ and will project the most significant number of cases (2). Over the years, it would increase rapidly than other regions and heavily economic burdensome due to lack of income and profoundly impact the social and health care system worldwide.

One of the critical approaches to help patients with AMD was visual rehabilitation with low-vision magnifiers, like spectacles, hand-held telescopes, and magnifiers (7). However, there are some drawbacks. Firstly, those extraocular devices restricted the visual fields (VFs) and are limited to the relative movement between the eye and telescope, causing patients to move their heads to follow the image (8). Secondly, they are cosmetically burdensome and lead to motion sickness because of vestibular-ocular conflict (9).

Given the drawbacks illustrated above, improving visual acuity (VA) and quality of life among AMD patients remains an ongoing challenge. Intraocular implants, therefore, as a visual prosthetic device, have been developed as attractive alternatives. Nevertheless, it is unknown which devices are more effective and safer. The previous major review in 2017 has illustrated the optical fundamentals of IOLs for AMD and compared seven intraocular lenses (10). The purpose of this systematic review is to compare the efficacy and safety of different intraocular lenses for patients with AMD based on updated evidence. We present the following article following the PRISMA reporting checklist (available at http://dx.doi.org/10.21037/atm-20-5851).

\section{Methods}

Two researchers (WJ and MFF) initially searched the Cochrane Library, PubMed using pre-reported search terms and keywords combined with both Mesh term and text words. The full-text versions of any study were then screened independently and discussed when disagreements were exited to avoid article selection bias. The search term includes (Macular degeneration [MeSH] OR Macular degeneration) and (intraocular lens OR visual devices OR implantable iol) OR EyeMax Mono OR iolAMD OR Scharioth lens OR Fresnel Prism OR IOL-VIP System
OR Implantable Miniature Telescope OR Lipshitz macular implant. We searched randomized clinical controlled trials, cohort studies no matter prospective and retrospective, and case serial reports published from 2017 to 2020 April. However, for some devices that lack updates, we further summarize their characteristics in three aspects: VA outcomes, quality of life and functional outcomes, and safety outcomes. The language is specific to English, and the only intraocular lens or device in human patients affected by AMD was identified as eligible studies to include in our review. Extraction data included the necessary information (principle of the equipment, fundamental parameters), characteristics of patients (number of patients, age, assessment methods for AMD, and indication criteria), surgical operation (size of the incision, implantation position, timing for operation, binocular or monocular, contraindications and adverse events), preoperative evaluation (exercises, prefer retinal location (PRL) and approaches to measure VA), outcomes (short-time and long-time efficacy) and characteristics of the studies (title, publication year, country, and study design). This study's primary outcome was the postoperative VA gain related to each device, and the secondary outcome was the evaluation of the quality of life, which is the original intention to design and promote those implantable visual devices.

\section{Results}

\section{Intraocular magnifier telescope (IMT)}

IMT is a device that designs for monocular use reduces the impact of the central vision blind spot. It projects the objects onto the larger area of the light-sensing retina that is not degenerated by AMD (11). It has comprised of high precision quartz glass wide-angle micro-optics mounted in a polymethyl methacrylate (PMMA) carrying device and, after removing the natural lenses, implanted in the capsular bag through a $10-12 \mathrm{~mm}$ limbal or scleral incision tunnel to extends through the pupil and acquire vertical clearance distance about $2.5 \mathrm{~mm}$ from the posterior cornea in case of damaging the posterior cornea endothelium The parameters of the device are $4.4 \mathrm{~mm}$ long, $3.6 \mathrm{~mm}$ in diameter, weigh $60 \mathrm{mg}$ in aqueous and the overall haptic-to-haptic diameter of $13.5 \mathrm{~mm}$. The lens aperture is $3.2 \mathrm{~mm}$. There are two forms depends on different magnifications, namely the WA (wide-angle) $2.2 \times$ and the WA 3.0× (FDA 2010).

The most common approach used in IMT was a fixed focus Galilean telescope system. Two optical elements 
Table 1 Comparison between WA-IMT model with NG (SI) system

\begin{tabular}{lll}
\hline Parameters & WA-IMT & NG $(\mathrm{Si})$ \\
\hline Magnification & $2.2 \times / 2.7 \times$ & Same \\
Optics diameter $(\mathrm{mm})$ & 3.60 & Same \\
AXL (height, $\mathrm{mm})$ & 4.40 & Same \\
Overall diameter $(\mathrm{mm})^{\star}$ & 13.5 & 10.8 \\
Incision size $(\mathrm{mm})$ & $10-12$ & $7.0-7.5$ \\
Capsulorhexis size $(\mathrm{mm})$ & 7 & 6 \\
ECD loss & $23-25 \%$ & $7.9 \%$ \\
Corneal clearance-post op ACD $(\mathrm{mm})$ & 2.5 & 3.5 \\
Sutures & $8-12$ & $3-4$ \\
Manipulation & High rate & Almost none \\
Surgical duration $(\mathrm{min})$ & 60 & 25 \\
Surgical procedure & For skilled surgeon & Simpler, less complicated \\
Patients feedback & Few weeks PO & Few days PO
\end{tabular}

* include the haptic loop. ECD, endothelium cell density; ACD, anterior chamber depth.

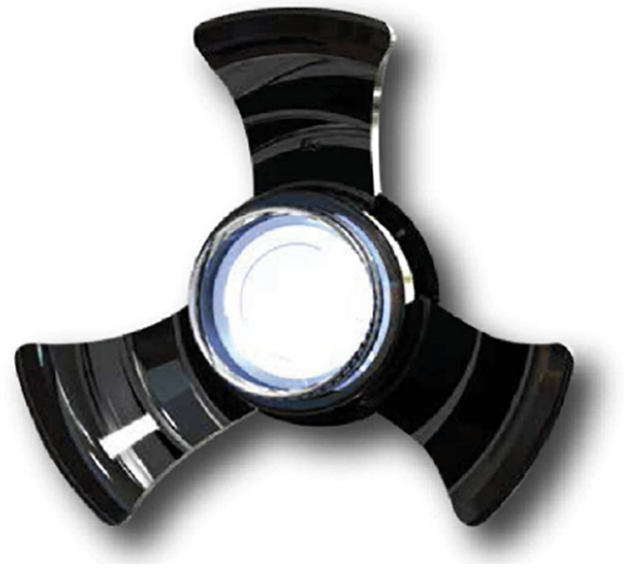

Figure 1 NG SI IMT 3X. It was supplied by the VisionCare Ophthalmic Technologies Company.

with high positive and negative power were used combined with the cornea embedded in the air to achieve higher magnification. The central VF of an operative eye would magnify $3 \times$ with a $20-24^{\circ}$ field of view projected onto about $55^{\circ}$ of the retina and the vision range, including near, intermediate, and distance. In contrast, the fellow eye is the sole eye for peripheral vision after surgery. However, the field of view is magnification at the expense of a significant VF reduction, which is not suitable for binocular implantation. Vision extensive rehabilitation for 6 months to 1 year were requested for all patients after surgery to be effectively using the device (12). With a low vision specialist's help, the recipients would learn to reteach the brain to learn how to use each eye differently for a specific task.

The US FDA first approved in 2010 and reduced the potential candidates' age limits from over 75 to over 65 years old in 2014. There are other limitations to FDA guidelines. The treatment candidate would have irreversible dry AMD, no longer for drug treatment, and no previous cataract surgery in the implanted eye (13). In 2020, the new models of IMT, namely WA $2.2 \times$, WA $3 \times$, WA DE $3 \times$, NG IMT, and Tsert SI (NG SI IMT $3 \times$ ), were CE marked and approved in European Union (Table 1). The new device (Figure 1), NG SI IMT $3 \times$, is supplied as preloaded in the Tsert SI cartridge (Figure 2) what allows the implantation with a much smaller incision (SI) than with the previous model (Figure 3).

There were eight articles eligible and identified (14-21), and 274 patients contributed to the efficacy and safety analysis; among them, 174 patients were participated in follow-up studies at 18 and 24 months, whereas 63 patients eventually finished the follow-up study at 60 months. 


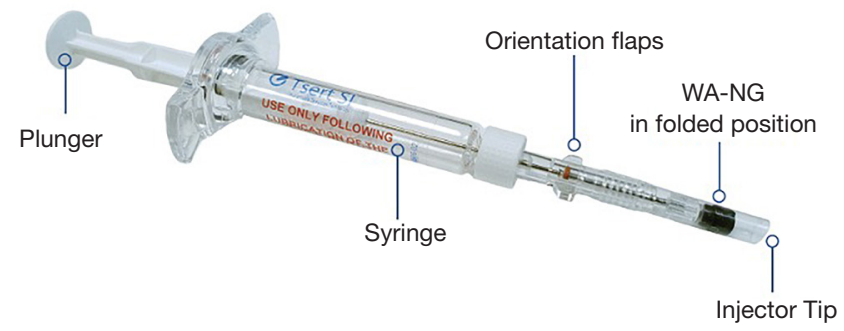

Figure 2 Tsert $\mathrm{Si}^{\mathrm{TM}}$ Loaded Injector. It was supplied by the VisionCare Ophthalmic Technologies Company.

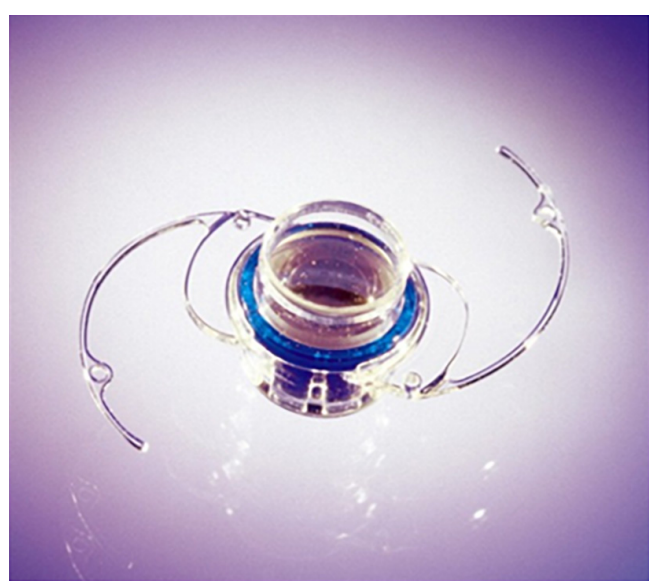

Figure 3 WA-IMT model. It was supplied by the VisionCare Ophthalmic Technologies Company.

\section{VA outcomes}

A prospective cohort study (14) includes 40 AMD patients (mean age was 77.1 years \pm 5.9 ), shows that both uncorrected distance visual acuity (UCDVA) and uncorrected near visual acuity (UCNVA) were improved by $0.3 \log$ MAR and 0.4 Log MAR respectively. Another 1-year clinical trial done by Lane (15) found out that $62 \%$ of participants gained three or more lines in both distances and near best-corrected visual acuity (BCVA), whereas $77 \%$ gained two or more lines. The most essential non-randomized multicenter casecontrol trial done by Hudson from 2006 to 2015 (16-18) shows that among 217 participants (mean age was 76 years old), $53 \%$ of operative eyes reached a 3 -line or more improvement in BCVDA and BCNVA. For the 2-year follow-up trial, there are 174 patients participated, and $59.5 \%$ gained 3 -line or more of BCVA. The increased percentage of VA improvement may occur due to the age of subjects may younger and satisfied with the operation results. In 2015, 63 patients were followed and divided into two groups based on their age. Group $1(\mathrm{n}=31)$ was included patients from 65 to 75 years old, and Group $2(\mathrm{n}=32)$ was 75 and over. In the younger group, 58\% retained 3-line or more after five years of operation, and only $38 \%$ improved in the elder group. The mean BCDVA has improved $2.41 \pm 2.69$ lines in all patients, lower than results at 2 years (3.2 lines). Only 1 patient (3.2\%) had lost $\geq 2$ lines of vision than $3(9.4 \%)$ in group 2. Comparing the different models of IMT in patients at 1 and 2 years all show the $3 \times$ device had more considerable improvement in BCDVA than those implanted with the $2.2 \times$ device $(3.6 \pm 1.9$ versus $2.8 \pm 2.3$ lines, $\mathrm{P}<0.001)$.

All research illustrated above shows that younger patients whose age is beyond 75 may have more motivation and thoughts to understand fully and participants to the vision of extensive rehabilitation, which is the primary role in improving visual acuity and used to the eyes have different tasks. Therefore, they would gain more improvement compared with the older one. It may notice that postoperative low-vision rehabilitation was painful for elderly patients and should be communicated with them ahead of the surgery.

\section{Quality of life and functional outcomes}

Three articles evaluate the quality of life improvement, which is one of the essential tasks for IMT implantation in patients with advanced or end-stage AMD. Activities of daily life (ADL) questionnaire applied in 2 articles $(15,16)$, which represents activities of daily living grouped by two variables: type of activity (static or dynamic) and focusing distance (distance, intermediate, or near).

It shows a mean 14.1-point difference improvement (41.4 to $55.8, \mathrm{P}<0.001$ ) with statistically significant for all vision range for both static and dynamic dimensions at 1 year (16). Moreover, patients with younger age and more significant improvement in BCVA tended to score higher (15). 
One study uses NEI VFQ-25 scores and shows the mean scores were improved by more than 7 points on 7 of 8 relevant subscales and correlated with a more significant point increase in BCDVA and BCNVA at least 2 lines improvement $(\mathrm{P}=0.0175)$ (15). However, the Peripheral Vision subscale decreased significantly from 67.6 to 62.9 $(\mathrm{P}<0.001)$, which is explainable that it would be a new experience to use one eye solely focus on the central area and the sensitivity in both eyes would be decreased (11). The effectiveness and cost-effectiveness of the IMT were evaluated by Brown (19) in 2011 by using quality-adjusted life-year (QALY) gain to quantify and be comparable. The incremental cost-utility ratios of $\$ 19,302 /$ QALY for IMT implantation versus $\$ 16,045 / \mathrm{QALY}$ for fellow eyes that underwent intra-study cataract extraction. The results suggest that IMT is also well within the conventional limits of incremental cost-effectiveness.

\section{Safety outcomes}

Safety outcomes were mainly pointing at the rate of complications or adverse effects (AEs) at different periods of following time after surgery. Although the earliest 3 cases serial study done by Kaşkaloğlu in 2001 (20) fail to provide enough evidence to show VA improvements, they still bring about the possible complications like mild iritis and persistent bubbles existing inside the IMT that proved by studies based on larger populations. The most common AE illustrated by multiple types of research is inflammatory deposits on the device from $17.5 \%$ to $42.9 \%$, and the rate increased as time goes by from $21 \%$ at 1 year to $25 \%$ at 2 years (15-17). Another common AE is Pigment deposits on the device from $10 \%$ at 1 year to $11 \%$ at 2 years $(16,17)$. The treatment-needed intraocular pressure (IOP) increasing within 7 days, detected in $28 \%$ of participants (16). The rate of corneal edema, like conventional cataract surgery, was higher in the elderly (7.1\% versus $4.3 \%)$ (18).

The IMT replaced were seen in $3.9 \%$ to $15 \%$ because of patient request, corneal edema, and the failure of the IMT manufacturer's safety precautions to cause bubbles inside existing $(14,17,18)$. The glare in bright light and reduced peripheral vision and depth perception reported being complained and drove dissatisfaction to the surgery outcomes (17). That remains that those improvements in the quality of manufacture and make strict patient selection criteria are the way to decrease the future incidence of such complications (14).

Although the design for IMT tried very hard to keep the device away from the posterior endothelium, the loss of endothelium cell density (ECD) was still reported by all researchers. The mean cell density decreased by $20 \%$ at 3 months, $13 \%$ to $25 \%$ at 1 year, and $29 \%$ at 2 years $(17,18)$. At 5 years, the mean ECD loss was up to $35-40 \%$ among patients over 65 (18). The ECD loss may be correlated to baseline anterior chamber depth (ACD). One study shows that patients with $3.0 \mathrm{~mm}$ or more ACD are losing less endothelium cell counts at 2 years comparing with ACD less than $3.0 \mathrm{~mm}(17)$.

One ongoing study in IMT compares the safety and effectiveness of implanting the IMT in pseudophakia patients and is also estimated to be done in 2020 (21). In the Future, IMT clinical outcomes evaluation would need more studies to compare individuals randomized to the device versus non-implanted severed as controls.

\section{IOL-VIP System (Intraocular Lens for Visually Impaired People) (Figure 4)}

Another magnifier Galilean type telescope is the IOL-VIP System, launched in 2001 consists of two special lenses. One is a high biconcave IOL (about -66 D) embedded in the capsular acted as the eyepiece, determining the magnification. Another one is a high biconvex IOL (about $+55 \mathrm{D})$ in the anterior chamber as an objective that can further modify the image. A new model called the IOLVIP Revolution has also been developed since April 2012, implanted in the capsular bag, unlike the classic form (22). Both IOLs are made of polymethyl methacrylate and can filter the ultraviolet light. The diameter of the two optics is $5 \mathrm{~mm}$, and the total length is $13 \mathrm{~mm}$, which can offer an estimated magnification for a distance of 1.3. After standard phacoemulsification, the thick IOL (axial thickness max is 1.5 for AC IOL and $1.5 \mathrm{~mm}$ for in-the-bag IOL) need further enlarge the corneal incision to $7 \mathrm{~mm}$ to facilitate the implantation. There is a software that can perform a functional evaluation of the objective and subjective Preferred Retinal Locus (PRL) and BCVA, contrast sensitivity, and reading speed.

Meanwhile, it can also be used for training the PRL before and after the surgery. One study shows that the IOL-VIP $^{\circledR}$ software would recognize more than $66 \%$ of patients whose PRL may too far from the fovea and/or not responding adequately to pre-surgical training ahead of the surgery so that none of the articles we found had reported an implantation removal (23). All patients were requested to undergo a 2-week preoperative training (12 30-min training sessions) and a 3 -month postoperative rehabilitation 

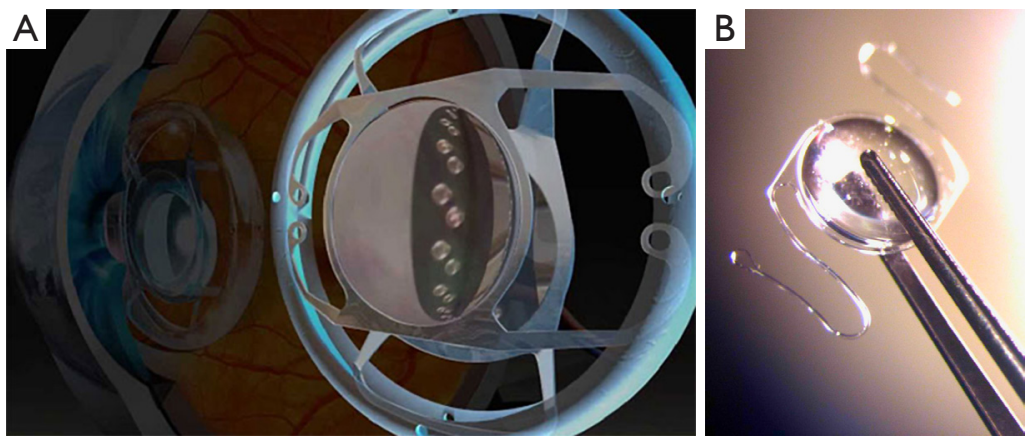

Figure 4 (A,B) Shows the IOL-VIP system, and Figure $4 A$ shows its position in the eye. It was supplied by LensSpecial Company.

program (5 30-min training sessions per week for 12) to train and consolidate the PRL (24).

There are 5 articles fitting into the evaluation of the efficacy and safety of IOL-VIP, and 107 patients in total participated in observational studies without any controls (22-27). Except for 1 article mainly discussed the IOL-VIP ${ }^{\circledR}$ software (23), others fit into the evaluation of the efficacy and safety of IOL-VIP. The mean follow-up time from 6 to 50 months.

A new study done by 2019 showed that 12 AMD patients with mean age 72.3 were enrolled, and BCVA pre- and post-operation were $1.08 \pm 0.14$ and $0.81 \pm 0.16 \log$ MAR and statistically significant between the difference $(\mathrm{P}=0.05)(25)$. Another noncomparative interventional case series (26) depicts the same improvements (the difference was 0.69 Log MAR), and the main best-corrected clinical gain was $44 \%$. Another case series study included 35 consecutive patients for 20 months (24). Besides the improvement in BCVA (difference was $0.51 \mathrm{Log}$ MAR), they also consider the reading magnification gain and postoperative reading distance gain, which was $\times 6.2$ and $7.66 \mathrm{~cm}$, respectively. To further evaluate the rehabilitation program's consequence, two groups were signed up by their preoperative VA. Patients with better preoperative VA tend to move their PRL to a more favorable position. In contrast, patients with low VA tend to have an unstable and peripheral PRL, which cannot be corrected by the training program. One retrospective study of 50 patients (age range between 36 and 85) with central scotoma for 4.2 years also detect the improvement in VA from 1.3 Log MAR to $0.68 \log$ MAR (27).

There is only 1 article (25) using VFQ-25 Scores for quality of life evaluation, and the difficulty with activities showed a statistically significant improvement from $39.4 \pm 16.3$ to $43.8 \pm 15.8$. Those improvements seen in scales may be related to the software using before the surgery, which can fitly evaluate patients' residual visual function with an accuracy of $78 \%$ and prognosis for visual improvement based on simulation of the postoperative condition and lower the patients' expectation. Therefore, a further study which including life quality evaluation and functional outcomes, would need to consider IOL-VIP System as more clinical applications.

The most common complication reported by studies is transient IOP increased after the operation. About $40 \%$ $(4 / 10)$ of patients had increased IOP $(24 \pm 3 \mathrm{mmHg})$ but can resolve in 1 week with the administration of topical timolol maleate $0.5 \%$ and corticosteroids (26). In one study, they do the preoperative iridotomy in all other cases after noticing 3 of the first 5 cases developed a pupillary block with IOP increased (24). Although 1 article done by Dag et al. (25) found that ACD was deeper post-operation from $2.7 \pm 0.3$ to $3.1 \pm 0.7$, the mean endothelium cell count was still significantly decreased postoperatively from $2,437 \pm 398$ to $1,849 \pm 376$, but lack of further information about the percentage among the subjects. Another study proved that endothelium cell loss appeared in $11.1 \%$ of subjects and $5.2 \%$ from 12 to 18 months (26). The incidence of PCO was $2.9-18 \%$ and can be managed by Nd: YAG laser capsulotomy $(24,27)$. All in all, IOL-VIP System has a high satisfaction by AMD patients with software that can simulate the postoperative VA improvement and fewer complications reported. However, lacking clinical controls makes those conclusions less evidential and needs more information to evaluate its efficacy and safety further.

\section{iolAMD}

The hyperopic acrylic iolAMD is another Galilean telescope based implant that consists of two lenses designed to an optimized retinal image to all areas of the macular 


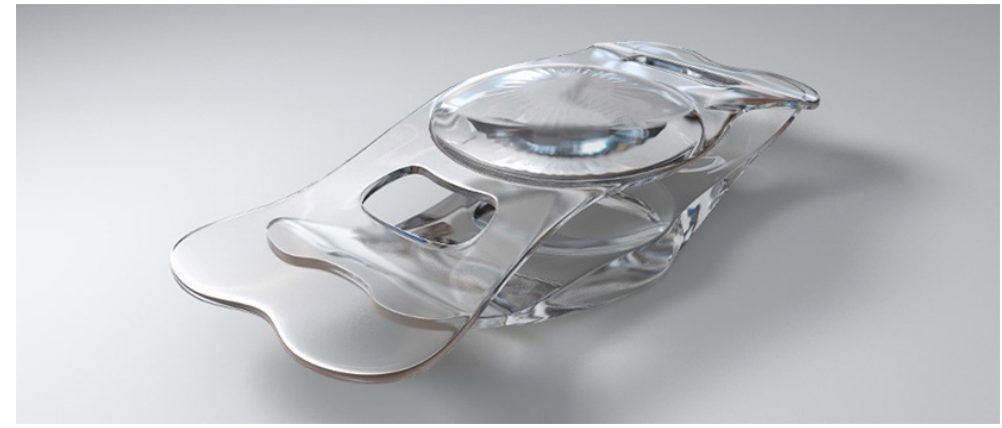

Figure 5 iol AMD. It was supplied by London Eye Hospital Pharma Company.

$\leq 10^{\circ}$ from fixation to 3 degrees away by a decentration of one of the IOLs and magnifies the image 1.25 to $1.3 \times$. IOL 1 is a high-plus power lens $(+63 \mathrm{D})$ implanted into the sulcus, which can gain depth of focus, whereas IOL 2 is a high-minus power lens (-49 D) embedded into the capsular bag. IOL 1 is $5 \mathrm{~mm}$, and the overall diameter is 11.75 to $12.0 \mathrm{~mm}$, with the haptic bent anteriorly. The optic of IOL 2 is $4 \mathrm{~mm}$, overall length is $11.0 \mathrm{~mm}$, and haptic vaulted posteriorly about $15^{\circ}$. After implantation, the distance recommended between optics of two lenses is $2 \mathrm{~mm}(28,29)$. The device can be folded, injected with a tip cartridge, and implant through a $3 \mathrm{~mm}$ non-suture incision without causing corneal astigmatism (30). The device's optics generate transverse asphericity and maintain a breadth of focus across the macula (31) (Figure 5).

Two case series studies were included $(29,31)$ with 15 patients participants, and none of the studies has controls and evaluates the quality of life-based on different scales.

For one case series study (31), whose only evaluated 3 patients with AMD, the mean CDVA was improved $0.40 \pm 0.17$ (from 0.08 to 0.5 ) and predicted CNVA was improved $0.49 \pm 0.13$ (from 0.15 to 0.6 ) for 1 month. Moreover, at the end of 3 months after the operation, the mean CDVA was improved to 0.6, and the CNVA remained stable. Another prospective intervention pilot study with 12 patients followed for 4 months (29) shows that the mean decimal CDVA gain 67\% (from 0.12 preoperatively to 0.20 ) and similar results in CNVA with less improvement about $50 \%$ (from 0.14 to 0.21 ). It also illustrated that the mean change in spherical equivalent (SE) was $-1.5 \mathrm{D}$ with a level of $0.5 \mathrm{D}$ of induced astigmatism, and it shows a myopic shift (from $-0.1 \mathrm{D}$ to $-1.6 \mathrm{D}$ after 4 months). Furthermore, the mean cylinder also improved. Microperimetry testing is also used to indicate a magnification effect and a deviation of images from the central of fovea up to $5^{\circ}$ with fixation stability. Meanwhile, the mean threshold sensitivity increased from 7.2 to $20.2 \mathrm{~dB}$. At baseline, the fixation points within a 2 -degree circle and a 4-degree circle were $31 \%$ and $71 \%$, respectively, whereas, after implantation, the number of points increased to $48 \%$ and $88 \%$.

Most studies recorded the incidence rate of IOP increasing. One study did a precautionary intraoperative iridectomy among 9 patients (12 patients in total), and there is only one patient whose IOP arouse from 14 to $22 \mathrm{mmHg}$ resolved by Nd:YAG laser (29). ECD reduced by $18 \%$ at 4 months, and the rate of corneal edema was $16.7 \%$, also reported by one study (29). One patient's IOL's in capsular bag vaulted anteriorly, which caused vision quality reduction and replaced with a smaller-size ordinary IOL. Except that, the other 11 patients checked by AS-OCT and found no evidence of gross deviation from the expected position (29).

Some demerits are existing in this device. First of all, two lenses combination restricted to eyes with previous cataract surgery and standard monofocal IOL implantation. Moreover, if the PRL changes with the progression of AMD, it would need further surgical manipulation, and patients usually use multiple PRLs for daily activities. A single restricted area would cause visual function damaging (30). Therefore, it is not offered by the manufacturer anymore today.

\section{EyeMax Mono}

According to the demerits of previous iolAMD devices, the advent of EyeMax Mono was approved in 2016 (32) and is similar to standard IOL, excepts for its optics that use hyperspherical design to increase the breadth of focus and the quality of the image supplied to all areas of the macula at $\leq 10^{\circ}$ of retinal eccentricity and magnifies the image $\times 1.1$ to $\times 1.2(33)$. One of the most advanced parts is 


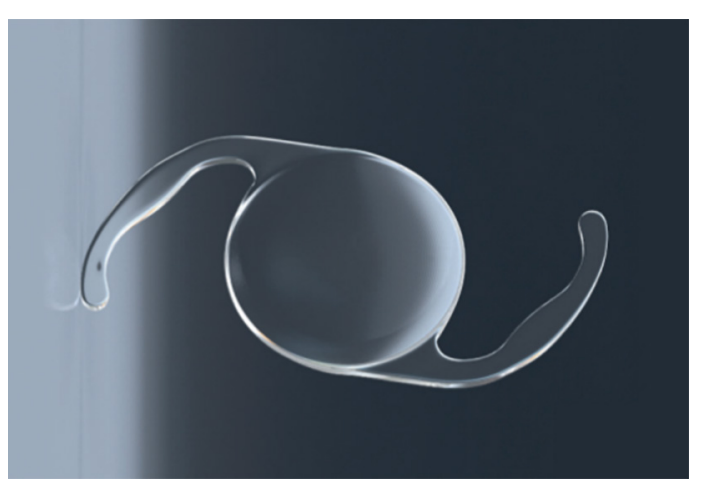

Figure 6 EyeMax Mono. It was supplied by London Eye Hospital Pharma Company.

to provide a high-quality image with reduced blur in those areas where photoreceptor cell densities may still afford VA of 6/30 Snellen or better. It's a new class of injectable, soft acrylic, extended macular vision IOLs (34) and permitting subjects to form single or multiple PRLs from their normal part of the macula as much as possible (35). There are two versions of the EyeMax mono: one is designed for capsular bag implantation following phacoemulsification. A second version is intended for sulcus implantation with standard, monofocal IOL that had already been implanted. The second version is a $-3.5 \mathrm{D}$ lens with a 5.0-mm hyperspherical optic component centered on a plate haptic component and delivers a modest hypermetropia correction. The overall diameter is 11.75 to $12 \mathrm{~mm}$, and the haptic is vaulted posteriorly to reduce the risks associated with sulcal IOL implantation. The implant requires a modest postoperative refractive target of $+2 \mathrm{D}$ to $+3 \mathrm{D}$ for up to $1.2 \mathrm{D}$ magnification (31). The EyeMax Mono can be binocularly implanted, and hypermetropia refractive target can be chosen to generate $10 \%$ to $20 \%$ magnification with spectacle correction in severe AMD cases (34) (Figure 6).

There were three consecutive cases of series studies included with 374 eyes. Robbie did the initial prospective interventional pilot assessment of the EyeMax Mono in 2018 (36), and 8 eyes of 7 patients with bilateral dry AMD underwent phacoemulsification and capsular bag implanted with 2 months follow-up postoperatively. Visual acuity improved for all patients, and mean CDVA improved from $0.93 \pm 0.22$ preoperatively to $0.59 \pm 0.25$, and the reading speed was reported a $57 \%$ improvement from $28 \pm 19$ to $44 \pm 31$ words per minute. Another prospective case series study included 22 pseudophakia eyes with 6 months followup (33). Both eyes of participants underwent small-incision sulcus implantation. The mean CDVA has improved $\geq 2$ lines in all participants' eyes. The evidence of improvement in CDVA over time from $0.90 \mathrm{Log}$ MAR preoperatively to $0.51 \mathrm{Log}$ MAR postoperatively at 6 months may suggest visual outcome improvement consists of a neuroadaptive process. Another consecutive case series of 244 eyes with mean age 80 years was conducted in 2018 and followed up for 3 months (37). Eighty-eight percent of cases reached the refraction target, and mean CDVA improved from 1.06 preoperatively to 0.71 postoperatively, equating to gain about 18 ETDRS letters. Mean CNVA was also improved from 1.36 to 0.88 postoperatively. Recently meta-analysis indicated that CDVA improvement with standard IOL implantation was only 6.5-7.5 ETDRS letters after 6-12 months of follow-up (38). In Austria, the first case report published in 2019 shows an 83-year-old Caucasian female with stable AMD in both eyes and did the operation on the left eye. It turns out after 6 months follow-up that her BCDVA improved from 0.2 to 0.5 (decimal), and BCNVA improved from 0.05 (decimal) to 0.2 (decimal) with no further long-and short-term complications (34).

The mean differences in endothelial cell counts were 143 , and $0.8 \%(2 / 244)$ experienced steroid-induced IOP increasing at 4 weeks after surgery, which can be under controlled on topical hypotensive drugs and quite the treatment of steroid treatment (37). Another study showed that $9 \%$ of subjects with pseudophakia eyes experienced IOP elevated after the surgery. So far, there was no report about diplopia and lens misposition (31).

Based on the evidence provided by case series studies, cataract extraction with EyeMax Mono implantation performed safely. It can benefit the subject's visual outcomes and optimized images across the macula. Other high-scale evidence levels with more subjects' studies would need to evaluate the safety, efficacy fully, and, most importantly, the quality of life and functional outcomes of this device.

\section{Scharioth macular lens (SML)}

The fundamental optical approach for this new add-on macular lens, SML, is near triad reflex (39). When patients focus on near objects with a constricted pupil, and light rays are restricted mainly to the central optical area, that would have a magnification effect. Still, when patients focus on distance with dilated pupil, light rays will pass through the peripheral regions, and it would not be influenced. The central optic region provides relatively high refraction to achieve sharp vision in a range of 10 to $15 \mathrm{~cm}$ from the 
eye. Cones that for color vision and VA concentrated in the fovea and Rods that are responsible for night vision, sensitive motion detection, and peripheral vision are abundant elsewhere in the retina. The reason why SML does not distribute light away from the fovea since we can detect motion and contours with non-foveal regions of the retina expect reading in the dark so that the device contributes to magnify the rest of the damaged fovea to enable reading.

The Scharioth macula lens (SML) is a one-piece foldable intraocular hydrophilic acrylic "add-on" lens with an overall diameter of $13 \mathrm{~mm}$ and a $1.5-\mathrm{mm}$ central portion with a refractive power of $+10 \mathrm{D}$ solely in pseudophakia eyes. It is implanted into the ciliary sulcus as an add on to the existing in-the-bag IOL through an incision of $2.2 \mathrm{~mm}$. It can be implanted simultaneously with standard phacoemulsification

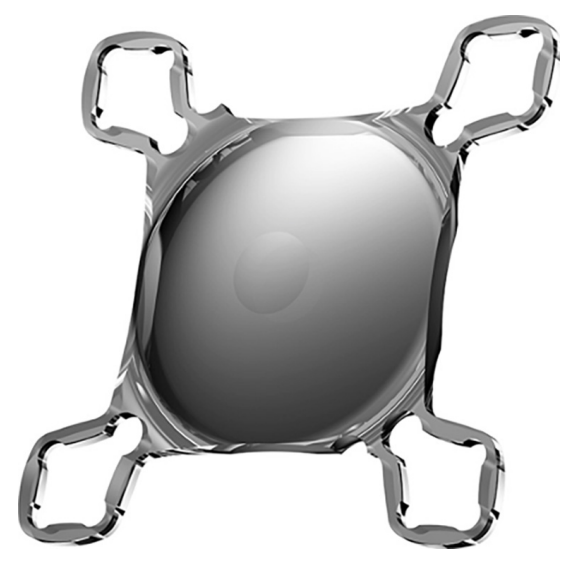

Figure 7 Scharioth macular lens. It was supplied by Medicontur Company. or at a late date (40). Wet AMD, iris neovascularization, progressive glaucoma, zonulopathy, and shallow ACD are not recommended for this device (39) (Figure 7).

There were 3 articles included in this review about SML, with 91 patients participated for follow-up from 3 to 12 months (41-43). In 2019, a prospective multicenter clinical trial done by Srinivasan reported the visual outcomes following monocular SML implantation in pseudophakia eyes with AMD (41). It includes 50 patients and a follow-up for 12 months. They only had CDVA of 0.4 to 0.1 (decimal), and CNVA can be improved about at least three lines when tested with a $+6.0 \mathrm{D}$ at reading distance of $15 \mathrm{~cm}$ and +2.5 D $40 \mathrm{~cm}$. The mean preoperative CNVA at $40 \mathrm{~cm}$ with $+2.5 \mathrm{D}$ addition was $0.23 \pm 0.12$ (decimal) preoperatively, and after 1 year, the UNVA at $15 \mathrm{~cm}$ can improve to $0.57 \pm 0.33$, similar as screening test measurement preoperatively. Furthermore, the mean CDVA remained unchanged at 1 year and confirmed that the SM does not affect the distance VA. No intro- and post complications have been reported. A retrospective study among 15 pseudophakia patients for follow-up 3 months also improved the CNVA and stable DVA (42).

Only one article presented in 2018 evaluated 26 patients' quality of life by VFQ-25 after SML implantation (43) showed $35 \%$ of patients achieved normal reading vision with a gain of at least 3 lines and $28 \%$ overall improvement on the questionnaire, especially for patients with legal blindness.

\section{Other approaches}

A new foldable one-piece high-add IOL called LENTIS ${ }^{\circledR}$
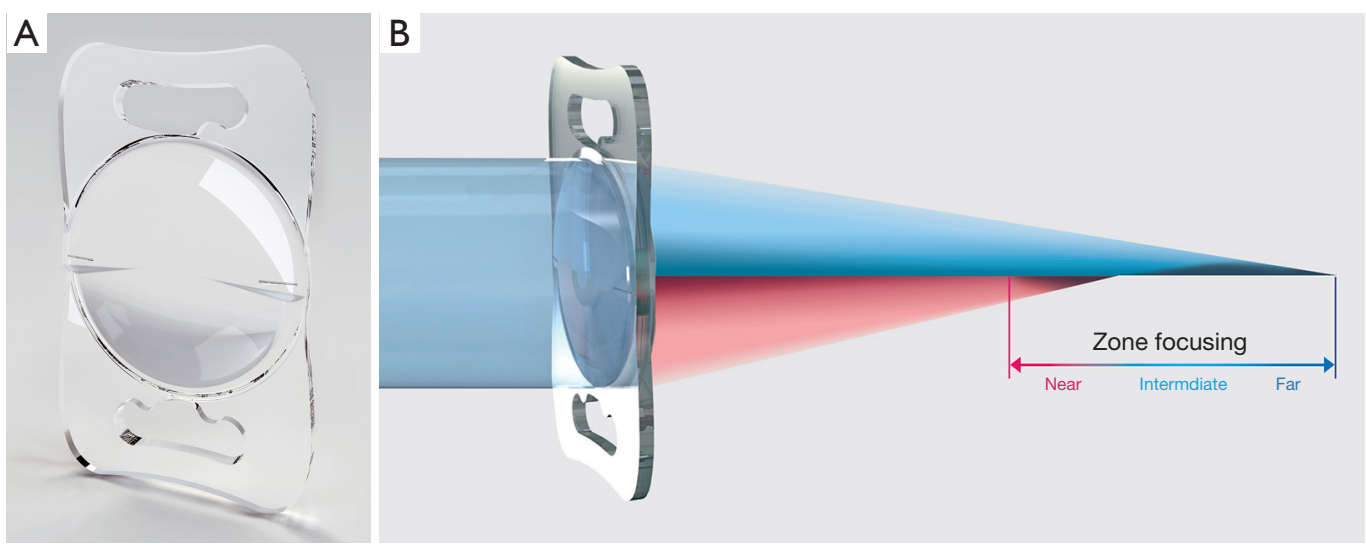

Figure 8 (A) LENTIS ${ }^{\circledR}$ MAX, LS-313 MF80 (has changed name to Oculentis); (B) shows paths of light rays. It was supplied by Teleon Surgical B. 
MAX, LS-313 MF80 (Teleon Surgical B.V, Berlin, Germany) (44) consisting of hydrophilic acrylates with a hydrophobic surface has been reported (Figure 8). IOL's overall diameter is 11 and $6 \mathrm{~mm}$ optic, which can be implanted through a $2.2 \mathrm{~mm}$ incision. The new feature is a second additional near segment on the lens's posterior surface, and the lens designed an aspheric biconvex that provides an additional power of $8.0 \mathrm{D}$, equating to $6.0 \mathrm{D}$ at the spectacle plane. The design of the lens can magnify $1.5 \times$ at a distance of $30 \mathrm{~cm}$ and $3 \times$ at $15 \mathrm{~cm}$. Moreover, a case report of an end-stage AMD patient implanted this highadd IOL was published and suggested improvement in both distances and near VA.

\section{Discussion}

There is no further evidence to evaluate the safety and efficacy of Fresnel Prism Intraocular Lens. As for Lipshitz macular implant (LMI) and LMI-SI, although there are also no further clinical studies, an ongoing randomized clinical trial to compare the OriLens intraocular telescope with standard low vision training in eyes with end-stage AMD (MIRROR 2017) (45). The results would expect in 2020. The essential characteristics and parameters about the lens discussed above had summarized in table implantable miniature telescope (IMT) was the only approved surgical device by FDA in the US and can provide central vision magnification at the cost of losing peripheral vision. Meanwhile, the incision for implantation is larger enough that it needs 6-8 sutures to close the wound and may cause severe astigmatism. IOL-VIP System and EyeMax Mono involves a smaller incision and be suitable for both phakic and pseudophakic eyes with similar magnification. Visual outcomes based on the restricted number of participants suggest that the VA improvement in EyeMax Mono is modest compared with IOL-VIP without extensive visual rehabilitation. The big breakthrough about EyeMax Mono was to provide a high-quality image in all macular areas extending up to $10^{\circ}$ from the fovea in case of PRL changes or AMD progression. And the improvement in visual acuity had been proved to be case series of 244 subjects. There is no doubt that a case-control study with quality of life and function evaluation would conduct further.

Recently, a prospective multicenter clinical trial about the SML in previous pseudophakic eyes with AMD was published in 2019 (41) and provided more information about the visual and refractive outcomes. Although the SML does not offer distance magnification, it can magnify $2 \times$ for objects in a range of $10-15 \mathrm{~cm}$ from the eye and can be implanted through a $2.2 \mathrm{~mm}$ incision with a reduction of postoperative astigmatism.

For validity and generalizability of the studies included, VA outcomes vary in nature so that comparisons of efficacy and safety are difficult. No randomized controls in each IOL except IMT. Others are all case series with restricted subjects and limited follow-up duration that would not provide enough convincing evidence. Moreover, most of the studies did not limit the age range in the inclusion criteria, and long-term evidence and quality of life evaluation are insufficient in most of IOL. Due to the long and complicated rehabilitation after operations, understanding, and adequate expectations from patients, especially endstage, should be informed ahead of time. In Table 2 we present characteristics of the significant intraocular devices available today for AMD patients.

All in all, those technological advancements in the treatment of AMD would bring new hope in VA and quality of life improvements to patients with no other treatment options previously. To date, more extensive, randomized, and long duration follow-up clinical trials are needed to further evaluation of safety and efficacy among different IOL based on other optic fundamentals. Based on the evidence so far, there are various strengths and limitations. Ophthalmologists' options should value multiple aspects about the ocular anatomy that thick IOL may cause pupil block and increased IOP, living habits especially for reading and costs for individual AMD patients with low vision. 


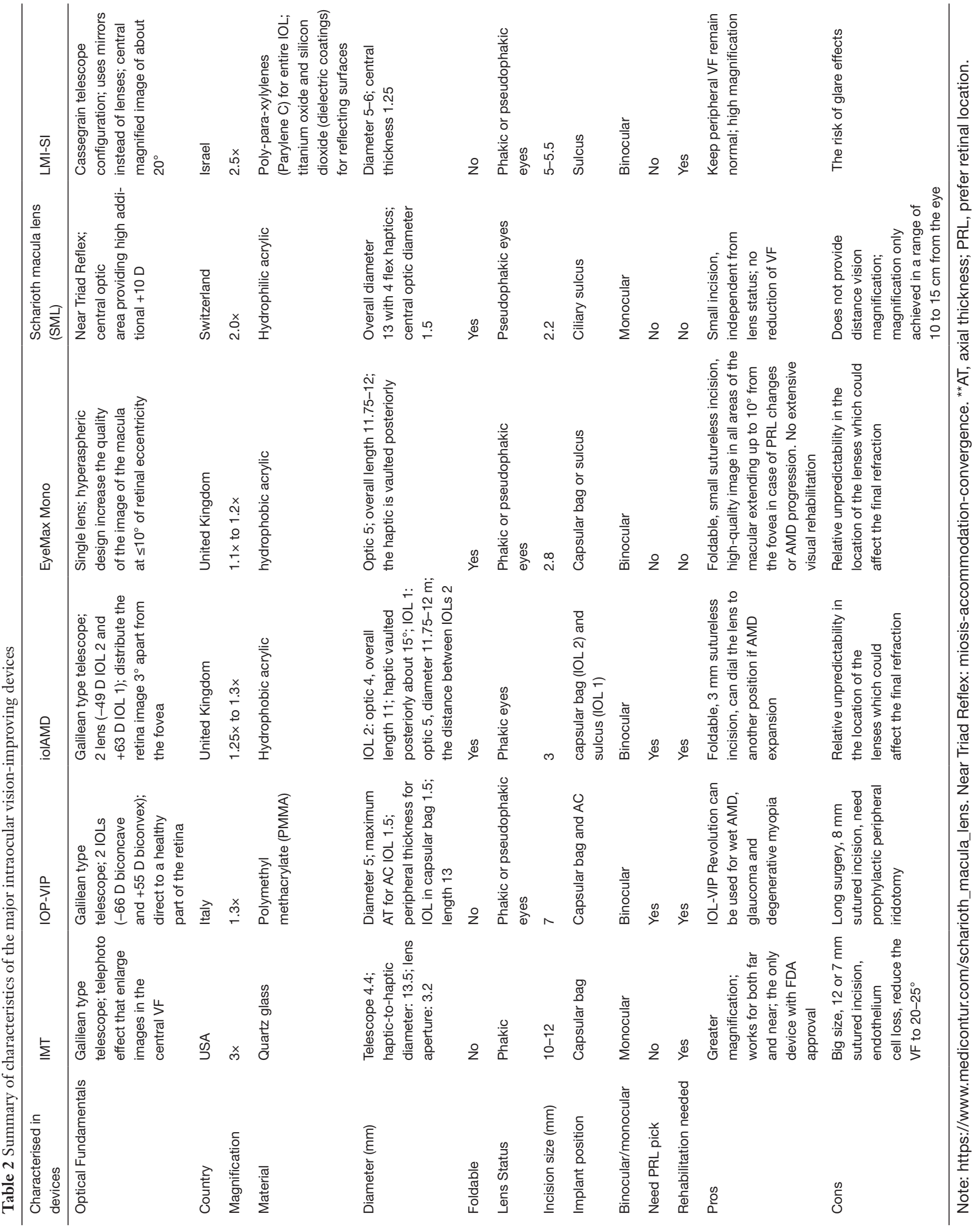




\section{Acknowledgments}

Funding: None.

\section{Footnote}

Provenance and Peer Review: This article was commissioned by the editorial office, Annals of Translational Medicine for the series "Recent Developments in Cataract Surgery". The article has undergone external peer review.

Reporting Checklist: The authors have completed the PRISMA reporting checklist. Available at http://dx.doi. org/10.21037/atm-20-5851

Conflicts of Interest: All authors have completed the ICMJE uniform disclosure form (available at http:// dx.doi.org/10.21037/atm-20-5851). The series "Recent Developments in Cataract Surgery" was commissioned by the editorial office without any funding or sponsorship. AG served as the unpaid Guest Editor of the series. The authors have no other conflicts of interest to declare.

Ethical Statement: The authors are accountable for all aspects of the work in ensuring that questions related to the accuracy or integrity of any part of the work are appropriately investigated and resolved.

Open Access Statement: This is an Open Access article distributed in accordance with the Creative Commons Attribution-NonCommercial-NoDerivs 4.0 International License (CC BY-NC-ND 4.0), which permits the noncommercial replication and distribution of the article with the strict proviso that no changes or edits are made and the original work is properly cited (including links to both the formal publication through the relevant DOI and the license). See: https://creativecommons.org/licenses/by-nc-nd/4.0/.

\section{References}

1. Mitchell P, Liew G, Gopinath B, et al. Age-related macular degeneration. Lancet 2018;392:1147-59.

2. Wong WL, Su X, Li X, et al. Global prevalence of age-related macular degeneration and disease burden projection for 2020 and 2040: a systematic review and meta-analysis. Lancet Glob Health 2014;2:e106-16.

3. Smith W, Assink J, Klein R, et al. Risk factors for agerelated macular degeneration: pooled findings from three continents. Ophthalmology 2001;108:697-704.

4. Joachim N, Mitchell P, Burlutsky G, et al. The incidence and progression of age-related macular degeneration over 15 years: The Blue Mountains Eye Study. Ophthalmology 2015;122:2482-9.

5. Mao F, Yang X, Yang K, et al. Six-Year Incidence and Risk Factors for Age-Related Macular Degeneration in a Rural Chinese Population: The Handan Eye Study. Invest Ophthalmol Vis Sci 2019;60:4966.

6. United Nations Population Division. World population prospects: the 2010 revision population database. Available online: http://esa.un.org/wpp/Documentation/WPP\%20 2010\%20publications.htm (accessed Nov 26, 2013).

7. Teh BL, Megaw R, Borooah S, et al. Optimizing cataract surgery in patients with age-related macular degeneration. Surv Ophthalmol 2017;62:346-56.

8. Tabernero J, Qureshi MA, Robbie SJ, et al. An aspheric intraocular telescope for agerelated macular degeneration patients. Biomed Opt Express 2015;6:1010-20.

9. Singer MA, Amir N, Herro A, et al. Improving quality of life in patients with end-stage age-related macular degeneration: Focus on miniature ocular implants. Clin Ophthalmol 2012;6:33-9.

10. Grzybowski A, Wasinska-Borowiec W, Alio JL, et al. Intraocular lenses in age-related macular degeneration. Graefes Arch Clin Exp Ophthalmol 2017;255:1687-96.

11. John Lazarou. Implantable Telescope Lens to Treat Macular Degeneration. Available online: Johns Hopkins. www. hopkinsmedicine.org/news/media/releases/implantable telescope_lens_to_treat_macular_degeneration_available_ at_johns_hopkins. Accessed 21 March 2013.

12. Dunbar HMP, Dhawahir-Scala FE. A Discussion of Commercially Available Intra-ocular Telescopic Implants for Patients with Age-Related Macular Degeneration. Ophthalmol Ther 2018;7:33-48.

13. Hau VS, London N, Dalton M. The treatment paradigm for the implantable miniature telescope. Ophthalmol Ther 2016;5:21-30.

14. Alió JL, Mulet EM, Ruiz JM, et al. Intraocular telescopic lens evaluation in patients with age-related macular degeneration. J Cataract Refract Surg 2004;30:1177-89.

15. Lane SS, Kuppermann BD, Fine IH, et al. A prospective multicenter clinical trial to evaluate the safety and effectiveness of the implantable miniature telescope. Am J Ophthalmol 2004;137:993-1001.

16. Hudson HL, Lane SS, Heier JS, et al. Implantable miniature telescope for the treatment of visual acuity loss due to end-stage age-related macular degeneration: one 
year results. Ophthalmology 2006;113:19872001

17. Hudson HL, Stulting RD, Heier JS, et al. IMT002 Study Group Implantable telescope for end-stage age-related macular degeneration. Long-term visual acuity and safety outcomes. Am J Ophthalmol 2008;146:664-73.

18. Boyer D, Freund KB, Regillo C, et al. Longterm (60-month) results for the implantable miniature telescope: efficacy and safety outcomes stratified by age in patients with end-stage age-related macular degeneration. Clin Ophthalmol 2015;9:1099-107.

19. Brown GC, Brown MM, Lieske HB, et al. Comparative effectiveness and cost-effectiveness of the implantable miniature telescope. Ophthalmology 2011;118:1834-43.

20. Kaşkaloğlu M, Uretmen O, Yagci A. Medium-term results of implantable miniaturized telescopes in eyes with agerelated macular degeneration. J Cataract Refract Surg 2001;27:1751-5.

21. The safety and effectiveness of implanting the IMT in pseudophakic patients. Registered at ClinicalTrials.gov: study number NCT03011554.

22. Dag MY, Afrashi F, Nalcaci S, et al. The efficacy of "IOLVip Revolution" telescopic intraocular lens in age-related macular degeneration cases with senile cataract. Eur J Ophthalmol 2019;29:615-20.

23. Savaresi G, Pierrottet CO, Orzalesi N. The IOL-Vip® Software: guidelines for IOL-Vip ${ }^{\circledR}$ implant. Invest Ophthalmol Vis Sci 2004;45:2010.

24. Orzalesi N, Pierrottet CO, Zenoni S, et al. The IOLVip System: a double intraocular lens implant for visual rehabilitation of patients with macular disease. Ophthalmology 2007;114:860-5.

25. Dag MY, Afrashi F, Nalcaci S, et al. The efficacy of "IOLVip Revolution" telescopic intraocular lens in age-related macular degeneration cases with senile cataract. Eur J Ophthalmol 2019;29:615-20.

26. Amselem L, Diaz-Llopis M, Felipe A, et al. Clinical magnification and residual refraction after implantation of a double intraocular lens system in patients with macular degeneration. J Cataract Refract Surg 2008;34:1571-7.

27. Six Years Experience With the IOL-Vip® Implant in Patients With Macular Degeneration. Available online: https://iovs.arvojournals.org/article. aspx? articleid $=2365600$

28. Hengerer FH. Sutureless telescopic IOL for patients with dry AMD. Cataract Refract Surg Today Eur 2015;2:40-1.

29. Qureshi MA, Robbie SJ, Tabernero J, et al. Injectable intraocular telescope: Pilot study. J Cataract Refract Surg 2015;41:2125-35
30. Lauren Lipuma (2015). The IOL can save sight, 2015.8 Available online: https://www.eyeworld.org/article-the-iolthat-can-save-sight

31. Hengerer FH, Artal P, Kohnen T, et al. Initial clinical results of a new telescopic IOL implanted in patients with dry age-related macular degeneration. J Refract Surg 2015;31:158-62.

32. Badala F, Bona E. A new extended macular vision intraocular lens for patients with cataract and macular disease: a consecutive case series; 37th ESCRS CongressParis 2019; Cataract Surgery Special Cases.

33. Hengerer FH, Auffarth GU, Robbie SJ, et al. First Results of a New Hyperaspheric Add-on Intraocular Lens Approach Implanted in Pseudophakic Patients with Age-Related Macular Degeneration. Ophthalmol Retina 2018;2:900-5.

34. Borkenstein AF, Borkenstein EM. A case report detailing use of a new intraocular lens with advanced technology, designed specifically for patients with center-involving macular disorders. Medicine (Baltimore) 2019;98:e16583.

35. Qureshi MA, Robbie SJ, Tabernero J, et al. Injectable intraocular telescope: Pilot study. J Cataract Refract Surg 2015;41:2125-35.

36. Robbie SJ, Tabernero J, Artal P, et al. Initial Clinical Results with a Novel Monofocal-Type Intraocular Lens for Extended Macular Vision in Patients with Macular Degeneration. J Refract Surg 2018;34:718-25.

37. Qureshi MA, Robbie SJ, Hengerer FH, et al. Consecutive case series of 244 age-related macular degeneration patients undergoing implantation with an extended macular vision IOL. Eur J Ophthalmol 2018;28:198-203.

38. Kessel L, Erngaard D, Flesner P, et al. Cataract surgery and age-related macular degeneration. An evidence-based update. Acta Ophthalmol 2015;93:593-600.

39. The magnifier in the eye, the new option for patients with macular disease. Available online: https://www.medicontur. com/scharioth_macula_lens

40. Scharioth GB. New add-on intraocular lens for patients with age-related macular degeneration. J Cataract Refract Surg 2015;41:1559-63.

41. Srinivasan S. Implantation of Scharioth macula lens in patients with age-related macular degeneration: results of a prospective European multicentre clinical trial. BMJ Open Ophthalmol 2019;4:e000322.

42. Bereczki Á. Experiences with the Scharioth Macula Lens new hope for patients with dry macular degeneration. Rom J Ophthalmol 2019;63:128-34.

43. Nielsen Niels V, Helgesen A, Muus G. Scharioth Macula 
Page 14 of 14

Lens for AMD: Quality of Life Study. Presented at: European Society of Cataract and Refractive Surgeons meeting, Vienna, Austria, September 22-26, 2018.

44. Borkenstein AF, Borkenstein E. Cataract surgery with implantation of a high-add intraocular lens LENTIS $®$ MAX LS-313 MF80 in end-stage, age-related macular

Cite this article as: Grzybowski A, Wang J, Mao F, Wang D, Wang N. Intraocular vision-improving devices in age-related macular degeneration. Ann Transl Med 2020;8(22):1549. doi: 10.21037/atm-20-5851

\section{Grzybowski et al. AMD intraocular vision improving devices}

degeneration: A case report of magnifying surgery. Clin Case Rep 2018;7:74-8.

45. MIRROR 2017 \{published data only\} How well does the OriLens (Hubble-type) implant work in improving vision in age-related macular degeneration? Available online: https://doi.org/10.1186/ISRCTN47403123 